\title{
Advanced memory effects in the aging of a polymer glass
}

\author{
L. Bellon, S. Ciliberto and C. Laroche \\ École Normale Supérieure de Lyon, Laboratoire de Physique, \\ C.N.R.S. UMR5672, \\ 46, Allée d'Italie, 69364 Lyon Cedex 07, France
}

November 16, 2018

\begin{abstract}
A new kind of memory effect on low frequency dielectric measurements on plexiglass (PMMA) is described. These measurements show that cooling and heating the sample at constant rate give an hysteretic dependence on temperature of the dielectric constant $\epsilon$. A temporary stop of cooling produces a downward relaxation of $\epsilon$. Two main features are observed i) when cooling is resumed $\epsilon$ goes back to the values obtained without the cooling stop (i.e. the low temperature state is independent of the cooling history) ii) upon reheating $\epsilon$ keeps the memory of all the cooling stops(Advanced memory). The dependence of this effect on frequency and on the cooling rate is analyzed. The memory deletion is studied too. Finally the results are compared with those of similar experiments done in spin glasses and with the famous experiments of Kovacs.
\end{abstract}

PACS: $75.10 . \mathrm{Nr}, 77.22 \mathrm{Gm}, 64.70 \mathrm{Pf}, 05.20-\mathrm{y}$. 


\section{1 introduction}

Glasses are out of equilibrium systems, which are characterized by a slow relaxation, which is often much longer than any laboratory scale. One of the main consequences is that after a quench into the glassy phase the properties of a glassy material depend on the time spent below the glass transition temperature $T_{g}$, that is to say the system ages.

The aging of glassy materials is a widely studied phenomenon [1, 2]. In spite of the interesting experimental [3, 4, 5, 6, 77, 8, 9, 10, 11, 12, 13] and theoretical progress [2, 14, 15, 16, 17], done in the last years, the physical mechanisms of aging are not yet fully understood. In fact on the basis of available experimental data it is very difficult to distinguish which is the most suitable theoretical approach for describing the aging processes of different materials. In order to give more insight into this problem several experimental procedures have been proposed and applied to the study of the aging of various materials, such as spin-glasses (SG) [3, 6, 7, 12, 13], orientational glasses (OG) [4, 8], polymers [1], 9, 10, 11, 18] and supercooled liquids (SL) 5].

The very well known Kovacs 18 experiment was probably among the first in showing that the state of a glass is strongly determined by its thermal history. In the Kovacs experiment the material is submitted to the following temperature cycle. The material is rapidly quenched from the liquid to the glass phase at a temperature $T_{1}$ at which the volume of the glass is $V_{1}$. Because of aging the volume of the material decreases as a function of time going from $V_{1}$ to $V_{2}$. Then the material is rapidly heated at a temperature $T_{2}$ at which $V_{2}$ is the equilibrium volume. One could think that no appreciable evolution appears as a function of time. Instead the volume first increases, reaches a maximum and then decreases to reach again the value $V_{2}$. The amplitude of the maximum depends of course on $T_{1}$. Another example of history dependent effect is that induced by the aging at low temperature, known as prepeak in polymer literature [9]. Such an aging does not produce important changes in the enthalpy. However when increasing temperature, a peak in the curve of the heat capacity versus temperature is observed at a temperature close to $T_{g}$.

Other procedures were proposed for spin glasses and applied to other materials. Among these procedures we may recall the applications of small temperature cycles to a sample during the aging time 3, 4, 5, 10. These experiments have shown three main results in different materials: i) there is an important difference between positive and negative cycles and the details 
of the response to these perturbations are material dependent [3], 田, 10]; ii) for SG [3] the time spent at the higher temperature sort of reinitialize the aging at a lower temperature whereas for plexiglass (PMMA) [10] and OG [4] it only slightly modifies the long time behavior; iii) A memory effect has been observed for negative cycles. Specifically when temperature goes back to the high temperature the system recovers its state before perturbation. In other words the time spent at low enough temperature does not contribute to the aging behavior at the higher temperature.

In order to have a better understanding of the free energy landscape of SG and OG, a new cooling protocol has been proposed [6] and used in several experiments [6, 7, 8]. This protocol, which is characterized by a temporary cooling stop, has revealed that in SG and in OG the low temperature state is independent of the complete cooling history but that these materials keep the memory of all the aging history (Memory effect) [6].

In a recent paper [11] we described an experiment where we have used the cooling protocol, proposed in ref.[6], to show that memory effects are present during the aging of the dielectric constant of plexiglass (PMMA), which is a polymer glass with $T_{g}=388 \mathrm{~K}$ [19]. The purpose of this article is to extend the results of ref. [11] and to show that more complex temperature cycle can be observed. The deletion effect is also discussed. Finally we compare the behavior of PMMA to that of SG and OG, submitted to the same cooling protocol.

As we already mentioned several kinds [9, 18] of "memory effects" for polymers have been described in literature. The most famous one is that of Kovacs [18. However the thermal cycles used in these experiments are quite different from those proposed in [6]. Thus it is interesting to check how polymers behave when they are submitted to this new thermal procedure, which can give new insight on the aging of these materials. Moreover, the use of the same protocols makes the comparison between different kinds of glasses easier.

The paper is organized as follows. In section 2 we describe the experimental set-up and we discuss the results of a single quench experiment. The aging properties of the PMMA dielectric constant are studied as a function of the quench temperature and of the measuring frequency. In section 3 the measure and the features of the memory effect are analyzed. In section 4 the memory deletion and the double memory effect are described. Finally in section 5 the results on the PMMA dielectric constant are discussed and compared with those observed in other systems. 


\section{Aging range: a simple quench experiment}

To determine the dielectric constant, we measure the complex impedance of a capacitor whose dielectric is the PMMA sample. In our experiment a disk of PMMA of diameter $10 \mathrm{~cm}$ and thickness $0.3 \mathrm{~mm}$ is inserted between the plates of a capacitor whose vacuum capacitance is $C_{o}=230 \mathrm{pF}$ (see fig. 1] for details). The capacitor is inside an oven whose temperature $T$ may be changed from $300 \mathrm{~K}$ to $500 \mathrm{~K}$. The temperature stability is within $0.1 \mathrm{~K}$. The maximum heating and cooling rate $|R|=|d T / d t|$ is about $180 \mathrm{~K} / \mathrm{h}$. We checked that the temperature difference between the two capacitor plates is always smaller than $1 K$ both during the heating and the cooling cycles.

The capacitor is a component of the feedback loop of a precision voltage amplifier whose input is connected to a signal generator. We obtain the real and imaginary part of the capacitor impedance by measuring the response of the amplifier to a sinusoidal input signal. This apparatus allows us to measure the real and imaginary part of the dielectric constant $\epsilon=\epsilon_{1}+i \epsilon_{2}$ as a function of temperature $T$, frequency $\nu$ and time $t$. Relative variations of $\epsilon$ smaller than $10^{-3}$ can be measured in all the frequency range used in this experiment, i.e. $0.1 \mathrm{~Hz}<\nu<100 \mathrm{~Hz}$. The following discussion will focus only on $\epsilon_{1}$ (thus we will omit subscript 1), for which we have the best accuracy, but the behavior of $\epsilon_{2}$ leads to the same conclusions.

The measurement is performed in the following way. We first initialize the PMMA history by heating the sample at a temperature $T_{\max }>T_{g}$. The sample is left at $T_{\max }=415 \mathrm{~K}$ for a few hours, so that equilibrium can be assumed for the initial condition. The temperature is rapidly decreased at $R=-180 \mathrm{~K} / \mathrm{h}$ to a temperature $T_{\text {stop }}<T_{g}$, where it is regulated by the oven. The zero of the aging time is taken at the instant, during the quench, when the sample temperature is equal to $T_{g}$. Typical aging curves of $\epsilon$ as a function of time are shown in fig.2, for different $T_{\text {stop }}$ at $\nu=1 \mathrm{~Hz}$ and different $\nu$ at $T_{\text {stop }}=365 \mathrm{~K}$. We clearly notice a logarithmic dependence on time of the dielectric constant as soon as the temperature is stabilized, in all the temperature and frequency range we have explored. However the aging curve depends on $T_{\text {stop }}$ and $\nu$ : the sample properties evolve faster when $T_{\text {stop }}$ is close to $T_{g}$ and $\nu$ is small.

Specifically one can write $\epsilon\left(T_{\text {stop }}, t, \nu\right)=A\left(T_{\text {stop }}, \nu\right)-B\left(T_{\text {stop }}, \nu\right) \log \left(t / t_{o}\right)$ with $t_{o}=1 h$. Here $A$ is the value of $\epsilon$ at $t=t_{o}$. It is found that $A$ and $B$ are functions of $T_{\text {stop }}$ and of the frequency $\nu$ at which the dielectric constant is measured. Note that as A and B depends also weakly on the cooling rate 
$R$, we always use $R=-180 \mathrm{~K} / \mathrm{h}$ in these experiments. The values of $B$, measured at $\nu=1 \mathrm{~Hz}$, are plotted in fig. (3) (a) as a function of $T_{\text {stop. }} . B$ is an increasing function of $T_{\text {stop }}$ till a temperature $T_{m}$ close to $T_{g}$, but goes down to 0 if $T_{\text {stop }}>T_{g}$. Indeed for a quench temperature larger than $T_{g}$ the sample can reach a thermodynamic equilibrium, so no aging is observed but only a relaxation of $\epsilon$ toward its stationary value. The long time behavior is stationary and $B$ goes to 0 .

The values of $B$, measured at $T_{\text {stop }}=365 \mathrm{~K}$, are plotted in fig.](b) as a function of $\nu . B$ is a slowly increasing function for $\nu \rightarrow 0$. Indeed aging is smaller at high frequencies than at low frequencies (a theoretical justification of such a behavior can be found for example in ref.[14]).

These curves determine the region where to work. In our dielectric measurement aging can be accurately observed between $335 \mathrm{~K}$ and $T_{g}=388 \mathrm{~K}$. We will probe $\nu=0.1 \mathrm{~Hz}$ and $\nu=1 \mathrm{~Hz}$ where aging effects are the largest in our frequency range.

\section{Simple memory}

In this section, we describe experiments following the protocols first proposed in ref. [6]: a temporary stop is done during the cooling of the sample, and its consequence on the dielectric constant behavior are studied. The measurement is performed in the following way: again, the PMMA history is first reinitialized by heating the sample at a temperature $T_{\max }>T_{g}$ and leaving it at $T_{\max }=415 \mathrm{~K}$ for a few hours. Then it is slowly cooled from $T_{\max }$ to a temperature $T_{\min }=313 \mathrm{~K}$ at the constant rate $R$ and heated back to $T_{\max }$ at the same $|R|$. The dependence of $\epsilon$ on $T$ obtained by cooling and heating the sample at a constant $|R|$, is called the reference curve $\epsilon_{r}$.

As an example of reference curve we plot in fig. (1)(a) $\epsilon_{r}$, measured at $0.1 \mathrm{~Hz}$ and at $|R|=20 K / h$. We see that $\epsilon_{r}$ presents a hysteresis between the cooling and the heating in the interval $350 K<T<405 K$. This hysteresis depends on the cooling and heating rates. Indeed, in fig.t(t)(b), the difference between the heating curve $\left(\epsilon_{r h}\right)$ and the cooling curve $\left(\epsilon_{r c}\right)$ is plotted as a function of $T$ for different $|R|$. The faster we change temperature, the bigger hysteresis we get. Furthermore the temperature of the hysteresis maximum is a few degrees above $T_{g}$, specifically at $T \approx 392 \mathrm{~K}$. The temperature of this maximum gets closer to $T_{g}$ when the rate is decreased.

We neglect for the moment the rate dependence of the hysteresis and 
we consider as reference curve the one, plotted in fig. obtained at $\nu=0.1 \mathrm{~Hz}$ and at $|R|=20 \mathrm{~K} / \mathrm{h}$. The evolution of $\epsilon$ can be quite different from $\epsilon_{r}$ if we use the temperature cycle proposed in ref. [6]. After a cooling at $R=-20 \mathrm{~K} / \mathrm{h}$ from $T_{\text {max }}$ to $T_{\text {stop }}=374 \mathrm{~K}$ the sample is maintained at $T_{\text {stop }}$ for $10 h$. After this time interval the sample is cooled again, at the same $R$, down to $T_{\min }$. Once the sample temperature reaches $T_{\min }$ the sample is heated again at $R=20 \mathrm{~K} / \mathrm{h}$ up to $T_{\max }$. The dependence of $\epsilon$ as a function of $T$, obtained when the sample is submitted to this temperature cycle with the cooling stop at $T_{\text {stop }}$, is called the memory curve $\epsilon_{m}$. In fig.5(a), $\epsilon_{m}$ (solid line), measured at $\nu=0.1 \mathrm{~Hz}$, is plotted as a function of $T$. The dashed line corresponds to the reference curve of fig. (a). We notice that $\epsilon_{m}$ relaxes downwards when cooling is stopped at $T_{\text {stop }}$ : this corresponds to the vertical line in fig.5(a) where $\epsilon_{m}$ departs from $\epsilon_{r}$. When cooling is resumed $\epsilon_{m}$ merges into $\epsilon_{r}$ for $T<340 \mathrm{~K}$. The aging at $T_{\text {stop }}$ has not influenced the result at low temperature. This effect has been called rejuvenation in recent papers 13, 14.

During the heating period the system reminds the aging at $T_{\text {stop }}$ (cooling stop) and for $340 K<T<395 K$ the evolution of $\epsilon_{m}$ is quite different from $\epsilon_{r}$. In order to clearly see this effect we divide $\epsilon_{m}$ in the cooling part $\epsilon_{m c}$ and the heating part $\epsilon_{m h}$. In fig. (b) we plot the difference between $\epsilon_{m}$ and $\epsilon_{r}$. Filled downwards arrows correspond to cooling $\left(\epsilon_{m c}-\epsilon_{r c}\right)$ and empty upward arrows to heating $\left(\epsilon_{m h}-\epsilon_{r h}\right)$. The difference between the evolutions corresponding to different cooling procedures is now quite clear. The system reminds its previous aging history when it is reheated from $T_{\text {min }}$. The amplitude of the memory corresponds well to the amplitude of the aging at $T_{\text {stop }}$ but the temperature of the maximum is shifted a few degrees above $T_{\text {stop }}$. On fig.6 we show that this temperature shift is independent of $T_{\text {stop }}$ for temperatures where aging can be measured in a reasonable time (above $335 K$ ). In contrast the amplitude of the downward relaxation at $T_{\text {stop }}$ is a decreasing function of $T_{\text {stop }}$, as expected from the first section measurements: no aging can be measured at $T_{\text {stop }}<335 \mathrm{~K}$ (see fig. 3(a)).

This memory effect seems to be permanent because it does not depend on the waiting time at $T_{\min }$. Indeed we performed several experiments in which we waited till $24 h$ at $T_{\min }$, before restarting heating, without noticing any change in the heating cycle. In contrast the amplitude and the position of the memory effect depend on $R$ and on the measuring frequency. As an example of frequency dependence, we compare in fig.7 two measurements done with the same $|R|=10 \mathrm{~K} / \mathrm{h}$ and waiting time $t_{\text {stop }}=10 \mathrm{~h}$ but for two 
different frequencies: $\nu=0.1 \mathrm{~Hz}$ and $\nu=1 \mathrm{~Hz}$. Again, as expected from the first section measurements, the higher is the frequency, the smaller is the downward relaxation, so the smaller is the memory effect. The positions of the maxima are at the same temperature.

As an example of rate dependence, at $\nu=0.1 \mathrm{~Hz}$ and $t_{\text {stop }}=10 \mathrm{~h}$, we plot in fig. 8 the difference $\epsilon_{m}-\epsilon_{r}$ as a function of $T$ for three different rates. The faster is the rate, the larger is the downward relaxation of the dielectric constant during the cooling stop. As the amplitude of the memory effect is equal to that of the relaxation, we just expect the memory effect to increase with $|R|$. But as we can see on fig. \&, the temperature positions of the maxima are rate dependent too: the larger is $|R|$, the farther the temperature of the maximum is shifted above the aging temperature $T_{\text {stop }}$. The cooling rate is not the only control parameter of the memory effect, the heating rate is relevant too.

\section{Advanced memory experiments}

In this section we apply to the PMMA sample more complicated temperature histories (inspired from spins glasses experiments [6, 12]): What happens if we try to read a memory twice? If we make two cooling stops ?

\subsection{Deleting memory}

In this experiment, we show that a memory can be read only one single time: reading a memory effect also deletes it. This experiment is inspired from similar ones done in spin glasses [12]. First we follow the classic procedure described in the previous section: during the cooling ramp at $R=-20 \mathrm{~K} / \mathrm{h}$, a temporary stop is done for $t_{\text {stop }}=20 \mathrm{~h}$ at $T_{\text {stop }}=345 \mathrm{~K}$, and then heating the sample from $T_{\min }$ with the same $|R|$. In fig.9(a) we plot the difference between the heating branches of $\epsilon_{m}$ and $\epsilon_{r}$ measured at $\nu=0.1 \mathrm{~Hz}$. The departure from the reference curve above $T_{\text {stop }}$ when lowering temperature is due to a smooth cutting of cooling, but this imperfection has no detectable effect on the heating curve. We can follow the memory of the cooling stop at $T_{\text {stop }}$, and when the memory curve almost merges the reference one, for $T_{i}=368 \mathrm{~K}$, we quickly stop heating and resume cooling at $R=-20 \mathrm{~K} / \mathrm{h}$. Notice that this inversion temperature $T_{i}$ is smaller than $T_{g}$, which means that the sample has not been reinitialized. When $T_{\min }$ is reached again, we 
make a classic heating at $R=+20 K / h$ (see the temperature history of the sample in the inset of fig.9(a)).

This second heating curve is really different from the first one: there are no tracks of the cooling stop at $T_{\text {stop }}=345 \mathrm{~K}$, but something like the memory of a stop around $370 K$. What we see now is in fact only the memory of the stop at $T_{i}$ : as the sample stays a few minutes around $T_{i}$ (the time needed to inverse $R$ to $-R$ ), it ages a little at $T_{i}$ and we find a memory of this event. This can be checked on fig.9(b), where we only show the memory of the inversion at the same temperature $T_{i}$, without the first memory. The curves corresponding to the second heating are exactly the same for the two experiments, showing that the first heating deletes all information about temperatures lower than $T_{i}$, even though $T_{i}<T_{g}$.

\subsection{Double memory}

More insight on the properties of the memory effects can be obtained by submitting the sample to a more complex cooling procedure consisting of two cooling stops. This procedure, which has been called the double memory effect, has been carried out successfully in spin glasses [6] where it has been observed that if two stops are done during cooling, the heating curve will present a memory effect for both stops. The difficulty that arise when trying to reproduce this experiment in PMMA is the narrowness of the aging range: when cooling the sample under $T_{\text {stop }}, \epsilon_{m}$ rejoins the reference curve only for temperatures where aging almost vanishes. It is therefore difficult to record two well distinct cooling stops. This is illustrated in fig.10, where $T_{\text {stop } 1}=375 \mathrm{~K}$ and $T_{\text {stop } 2}=345 \mathrm{~K}: \epsilon_{m}$ has not completely merged into $\epsilon_{r}$ when we stop cooling for the second time.

The double memory experiments allows us to point out another important property of PMMA aging: when temperature is lowered after the first stop, the system not only recover the same value of $\epsilon$, but also the same aging properties. Indeed, a $10 \mathrm{~h}$ stop at $345 \mathrm{~K}$ produces the same downward relaxation of $\epsilon$, whatever the previous history is. The low temperature state is thus completely uninfluenced by the high temperature history.

If we now heat the sample after the two cooling stops, we obtain the bolt curve of fig.11. If the memory of $T_{\text {stop } 1}$ is obvious, the lower temperature stop at $T_{\text {stop } 2}$ must be hidden in the first part of the curve. In order to check the presence of the memory effect of the second stop, let us suppose that this effect is just additive, that is the memory of a double stop is just the sum 
of the memory of both individual stops if their temperature is sufficiently different. So we plot with a dashed line in fig.11 the sum of the single memories of $T_{\text {stop } 1}$ and $T_{\text {stop } 2}$. Within errors bars, the two curves are the same, so we conclude that even if the memory effects overlap in the narrow aging range of PMMA, double memory experiments also work in this polymer.

\section{Discussion and conclusions}

We have applied to a PMMA sample the same temperature cycle used to study memory and rejuvenation effects in various kinds of spin glasses and in orientational glasses. Our dielectric measurements clearly show the presence of rejuvenation and memory in PMMA. As we already mentioned in the introduction the observation of a memory of the thermal history in PMMA is not new. However this memory effect has been obtained with a thermal protocol which is quite different from the one described in this paper. Thus the application to a polymer glass of the same procedure used in OG and SG allow us to address the question of the universality of the memory and rejuvenation phenomena in different materials. This is a very important point in to understand whether the same theoretical approach can be used to describe aging phenomena in different materials.

Let us summarize the main results of these low frequency dielectric measurements on PMMA:

a) The reference curve $\epsilon_{r}$, obtained at constant cooling and heating rate $|R|$ is hysteretic. This hysteresis is maximum a few degrees above $T_{g}$.

b) The hysteresis of $\epsilon_{r}$ increases with $|R|$.

c) Writing memory: a cooling stop produces a downward relaxation of $\epsilon_{m}$. The amplitude of this downward relaxation depends on $T_{\text {stop }}$ and it decreases for decreasing $T_{\text {stop }}$. It almost disappears for $T_{\text {stop }}<335 \mathrm{~K}$.

d) When cooling is resumed $\epsilon$ goes back to the cooling branch of the reference curve. This suggests that the low temperature state is independent on the cooling history.

e) Reading memory: upon reheating $\epsilon_{m}$ reminds the aging history and the cooling stop (Memory). The maximum of the memory effect is obtained a few degrees above $T_{\text {stop }}$. 
f) The memory effect does not depend on the waiting time at low temperature but it depends both on the cooling and heating rates. The memory effect increases with $|R|$.

g) Double memory effects are observed with more difficulty in PMMA than in SG. The difference comes from the fact that aging effects are reduced when temperature is lowered. However a careful analysis of experimental data allows us to show the existence of double memory effects in PMMA.

h) The memory effect is deleted by a reading, even if the temperature remains smaller than $T_{g}$.

Analogies between point a-b) for the hysteresis and point e-f) for the rate dependence of the memory effect leads to a new interpretation of hysteresis, which can be seen as the memory of aging at a temperature $T_{\text {stop }} \approx T_{g}$. Indeed in a free energy landscape model, a sample, which is cooled just above $T_{g}$, is in its equilibrium phase, that is in a favorable configuration at this temperature. If this configuration is not strongly modified by aging at lower temperatures then, when heating back to $T_{g}$, the system reminds this favorable state, just as it does in the memory effect.

It is interesting to discuss the analogies and the differences between this experiment and similar ones performed on SG [6, 7, 13] and on OG [8]. It turns out that, neglecting the hysteresis of the reference curve of PMMA and of OG, the behavior of these materials is quite similar to that of SG. A strong rate dependence has been observed in Ising spin glasses too [13]. During the heating period PMMA, SG and OG remind their aging history, although the precise way, in which history is remembered, is material dependent. Furthermore in these materials the low temperature state is independent on the cooling history. One can estimate the temperature range $\delta T$ where the material response is different from that of the reference curve because of the cooling stop. It turns out that the ratio $\delta T / T_{G}$ is roughly the same in PMMA, in SG and in OG, specifically $\delta T / T_{G} \simeq 0.2$. The important difference between SG and PMMA is in the dependence on $T_{\text {stop }}$ of the amplitude of the downward relaxation: it is strong in PMMA and weak in SG.

Our results seem to indicate that memory and rejuvenation phenomena in the aging process may be described by models based on a hierarchical free energy landscape, whose barriers grow when temperature is lowered [3, [6]. However the dependence of the memory effect on $|R|$ and the independence 
on the waiting time at $T_{\text {min }}$ mean that, at least for PMMA, the free energy landscape has to depend not only on temperature but also on $|R|$. Many models [3, 15, 20, 21] and numerical simulations [22, 23] do not take into account this dependence because they consider just a static temperature after a quench. In contrast point f) indicates that the cooling history is relevant too.

These difficulties can be avoided if one considers models based on a slow domain growth and domain walls reconformations in the pinning field created by disorder (see for example ref.[16] and references therein). These models imply the existence of a hierarchy of length scales $l$ with a characteristic time $\tau \propto l^{z}$. Recent numerical simulations [24] show that this is an important ingredient in order to have memory/rejuvenation effects. However one of the drawbacks of such a model is that memory is recovered only if the time spent at low temperature is short enough [16]. This effect does not seem to be true for PMMA, at least on reasonable laboratory time scales.

As a conclusion the "memory" and rejuvenation effects seem to be two universal features of aging whereas the hysteresis is present in PMMA and in $\mathrm{OG}$ but not in all kinds of spin glasses. It would be interesting to know whether these effects are observed in other polymers and in supercooled liquids, and whether the hysteresis interpretation in terms of a memory effect could hold for other materials. These measurements show that many features of aging seem to be "universal" in several materials and that models based on spin glasses may be useful to describe aging in polymeric materials.

\section{Acknowledgements}

We acknowledge useful discussions with J. P. Bouchaud, J. Kurchan, M. Mézard, G. Vigier and E. Vincent. We thank P. Metz and L. Renaudin for technical support. This work has been partially supported by the Région Rhône-Alpes contract "Programme Thématique: Vieillissement des matériaux amorphes" . 


\section{References}

[1] L.C. Struick, Physical aging in amorphous polymers and other materials (Elsevier, Amsterdam, 1978).

[2] Spin Glasses and Random Fields, edit by A. P. Young, Series on Directions in Condensed Matter Physics Vol.12 ( World Scientific, Singapore 1998).

[3] M. Lederman, R. Orbach, J.M. Hammann, M. Ocio, E. Vincent, Phys. Rev. B, 44, 7403 (1991); E. Vincent, J. P. Bouchaud, J. Hammann, F. Lefloch, Phil. Mag. B, 71, 489 (1995). ; C. Djuberg, K. Jonason, P. Nordblad, cond-mat/9810314.

[4] F. Alberici, P. Doussineau, A. Levelut Europhysics Lett. 39, 329 (1997).

[5] R. L. Leheny, S. R. Nagel, Phys. Rev.B 57, 5154 (1998).

[6] K.Jonason, E. Vincent, J. Hamman, J. P. Bouchaud, P. Nordblad, Phys. Rev. Lett. 81, 3243 (1998).

[7] T. Jonsson, K. Jonason, P. Nordblad, Phys. Rev. B 59, 9402 (1999); T. Jonsson, K. Jonason, P. Jonsson, P. Nordblad, Phys Rev. B 59,8770 (1999).

[8] P. Doussineau, T. Lacerda-Aroso, A. Levelut, Europhys. Lett., 46, 401 (1999).

[9] E. Muzeau, G. Vigier, R. Vassoille and J. Perez, Polymer, 36, (1995), 611.

[10] L. Bellon, S. Ciliberto, C. Laroche, cond-mat/9905160.

[11] L. Bellon, C. Laroche, S. Ciliberto, Europhys. Lett. 51, 551 (2000)

[12] K. Jonason, P. Nordblad, E. Vincent, J. Hammann and J.-P. Bouchaud, Eur. Phys. J. B. 13, 99 (2000)

[13] V. Dupuis, E. Vincent, J.P. Bouchaud, J. Hammann, A. Ito, H. Aruga Katori, Aging, rejuvenation and memory effects in Ising and Heisenberg spin glasses. cond-mat/0104399 
[14] J. P. Bouchaud, L.F. Cugliandolo, J. Kurchan, M. Mézard, in Spin Glasses and Random Fields, [2], and references therein.

[15] M.Mézard, G. Parisi, M. A. Virasoro, in Spin Glasses Theory and Beyond, World Scientific Lecture Notes in Physics Vol.9 ( World Scientific, Singapore 1987).

[16] J. P. Bouchaud," Aging in glassy systems: new experiments, simple models and open questions ", cond-mat/9910387 and 'Soft and Fragile Matter: Nonequilibrium Dynamics, Metastability and Flow', M. E. Cates and M. R. Evans, Eds., IOP Publishing (Bristol and Philadelphia) 2000, pp 285-304

[17] L. F. Cugliandolo, J. Kurchan, cond-mat/9812229 (1998)

[18] A. Kovacs, J. Polym. Sci. 30 (1958) 131.

[19] N. G. McCrum, B. E. Read, G. Williams Anelastic and Dielectric Effects in Polymeric Solids,(Dover 1991)

[20] D. S. Fisher, D. A. Huse, Phys. Rev. Lett. 56, 1601, (1987).

[21] A. J. Bray, M. A. Moore, Phys. Rev. Lett. 58, 57 (1987).

[22] E. Marinari, G. Parisi, J.J. Ruiz-Lorenzo, F. Ritort Phys. Rev. Lett. 76, 843 (1996); E. Marinari, G. Parisi J.J. Ruiz-Lorenzo, in Spin Glasses and Random Fields pp.59-98; E. Marinari, G. Parisi, J. J. Ruiz-Lorenzo Phys. Rev. B. 58, 14852 (1998).

[23] W. Kob, J.L. Barrat, Phys. Rev. Lett. 78, 4581 (1997).

[24] L. Berthier, P. C. Holdsworth, cond-mat/0109169v1 


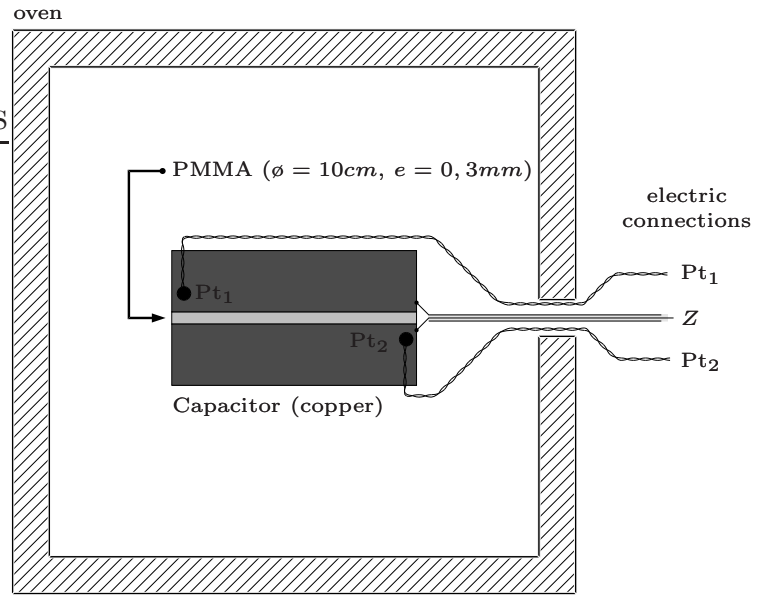

Figure 1: Experimental set-up

PMMA is the dielectric of a capacitor whose vacuum capacitance is $C_{0}=$ $230 \mathrm{pF}$. This impedance $Z$ is inside an oven which controls the temperature and acts like an electrical screen. The dielectric temperature is measured by two platinum probes $(\mathrm{Pt})$ 

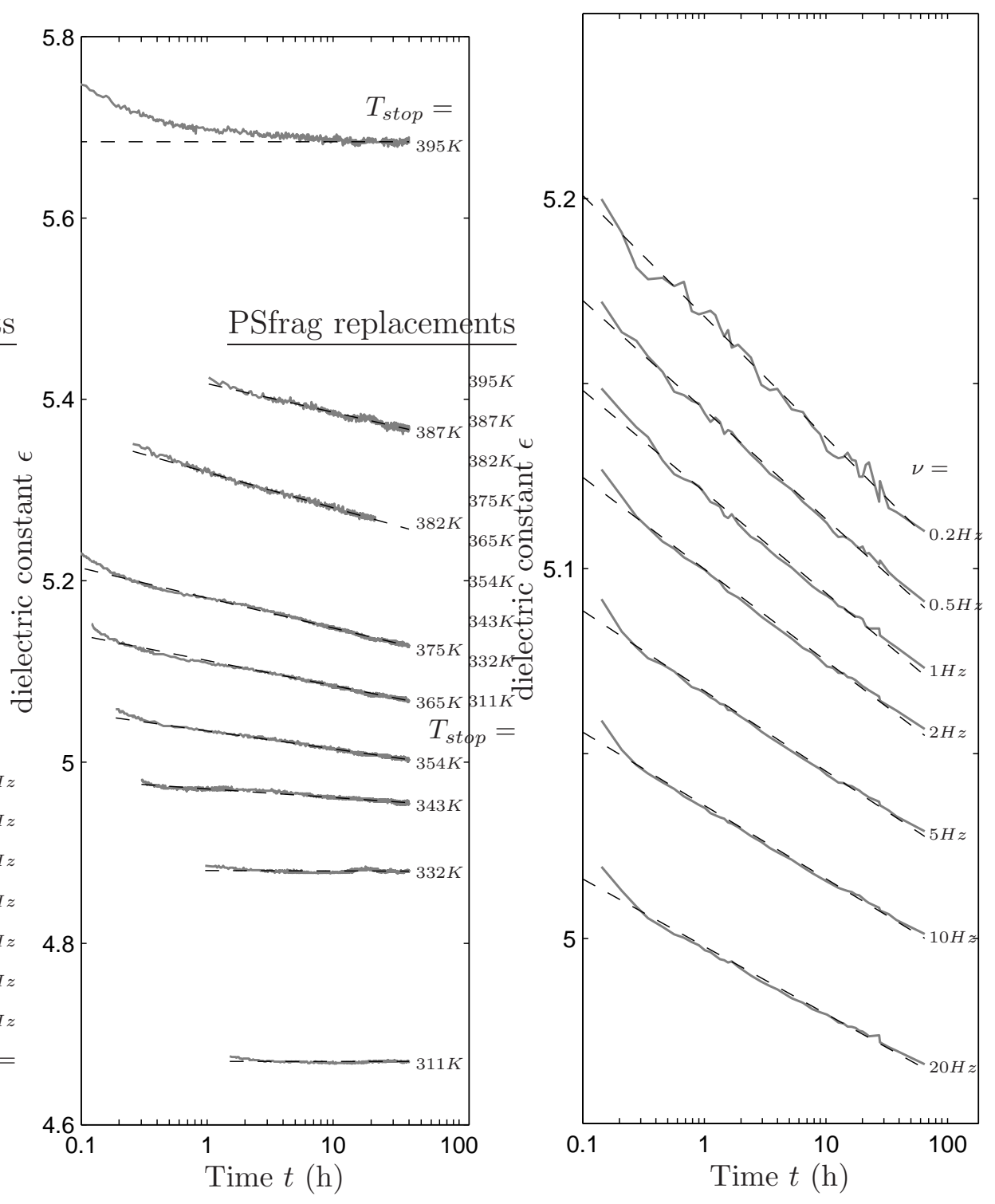

Figure 2: Dependence on $t$ of $\epsilon$ after a quench. (a) Aging measured at $\nu=1 \mathrm{~Hz}$ after a quench at various $T_{\text {stop }}$. (b) Aging measured after a quench at $T_{\text {stop }}=365 \mathrm{~K}$ at various $\nu$. A logarithmic fit in time of all these curves is accurate as soon as temperature is stabilized, except for $T_{\text {stop }}=395 \mathrm{~K}>T_{g}$ 

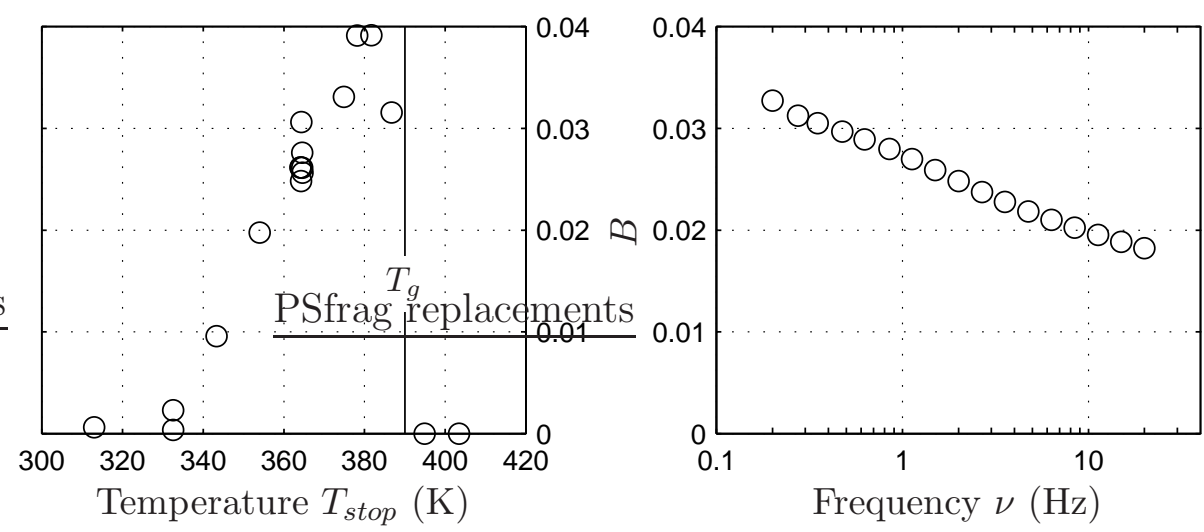

Figure 3: (a) Dependence on $T_{\text {stop }}$ of $B$ at $\nu=1 H z$, where $B$ is the parameter of the logarithmic fit of the dielectric constant $\epsilon\left(T_{\text {stop }}, t, \nu\right)=A\left(T_{\text {stop }}, \nu\right)-$ $B\left(T_{\text {stop }}, \nu\right) \log (t / 1 h)$ after a quench at $T_{\text {stop }}$. (b) Dependence on $\nu$ of $B$ at $T_{\text {stop }}=365 \mathrm{~K}$. 

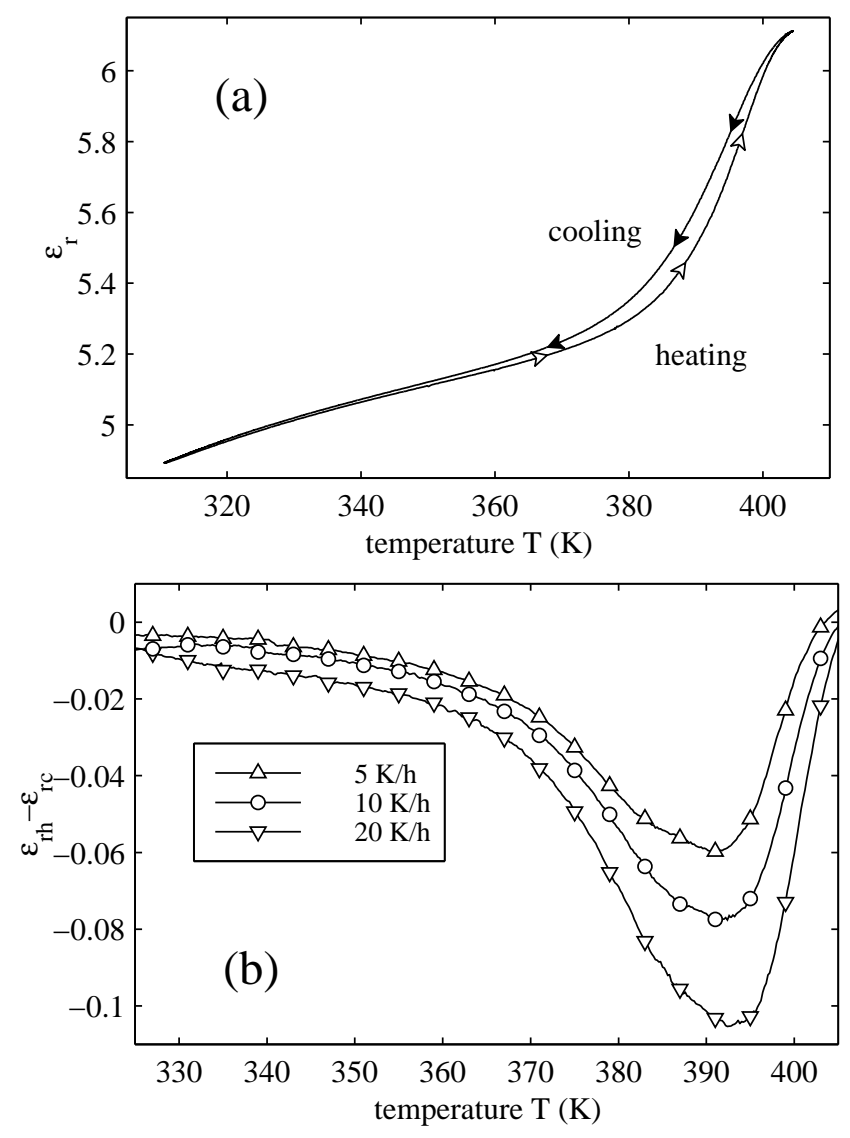

Figure 4: (a) Evolution of $\epsilon_{r}$ at $\nu=0.1 H z$ as a function of $T$. Reference curve obtained with $|R|=20 K / h$. (b) Hysteresis of the reference curve (difference between the heating and cooling curves $\epsilon_{r h}-\epsilon_{r c}$ ) for 3 different $|R|: 5 K / h(\triangle), 10 K / h(\circ)$ and $20 K / h(\nabla)$. 

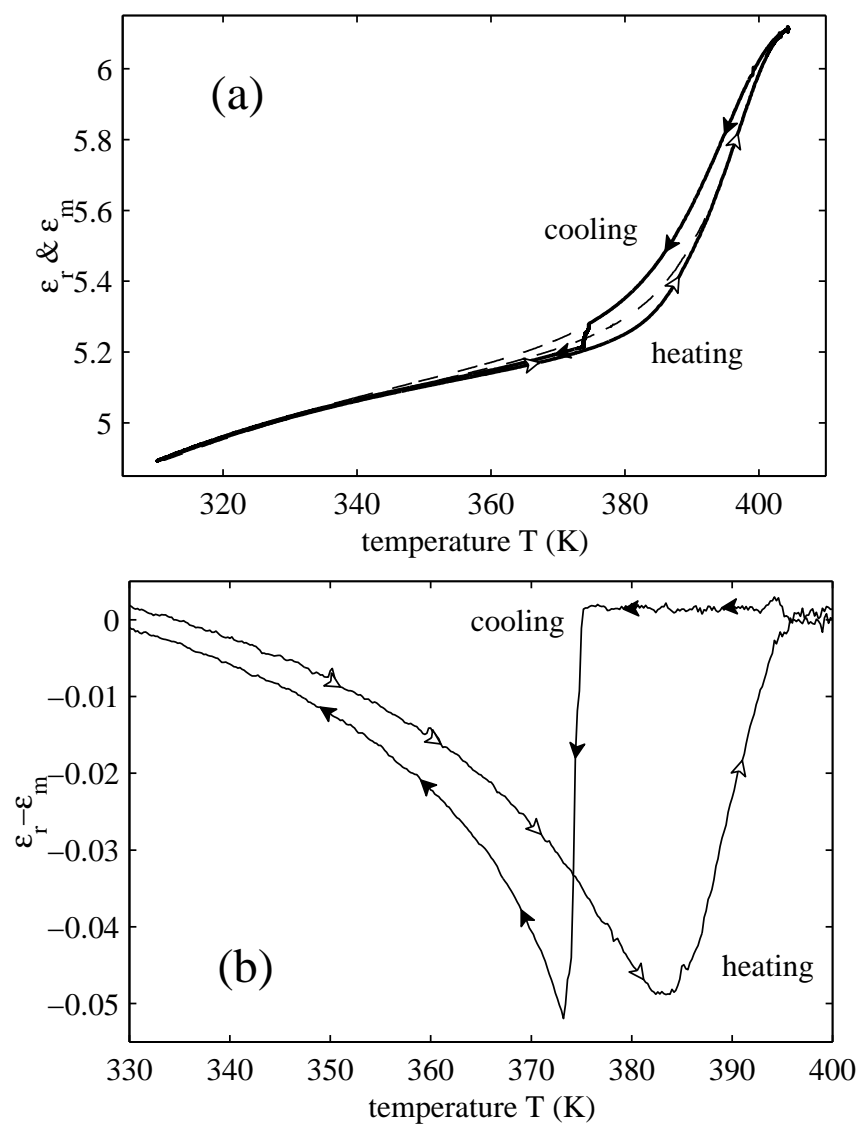

Figure 5: (a)Evolution of $\epsilon$ at $\nu=0.1 \mathrm{~Hz}$ as a function of $T$. The dashed line corresponds to the reference curve $\left(\epsilon_{r}\right)$ of Fig.t(a). The solid bold line corresponds to a different cooling procedure: the sample is cooled, at $R=$ $-20 \mathrm{~K} / \mathrm{h}$, from $T_{\max }$ to $T_{\text {stop }}=374 \mathrm{~K}$, where cooling is stopped for $10 \mathrm{~h}$. Afterwards the sample is cooled at the same $R$ till $T_{\min }$ and then heated again at $R=20 \mathrm{~K} / \mathrm{h}$ till $T_{\max }$. (b) Difference between the evolution of $\epsilon_{r}$ and $\epsilon_{m}$. Downward filled arrows correspond to cooling $\left(\epsilon_{m c}-\epsilon_{r c}\right)$ and upward empty arrows to heating $\left(\epsilon_{m h}-\epsilon_{r h}\right)$. 

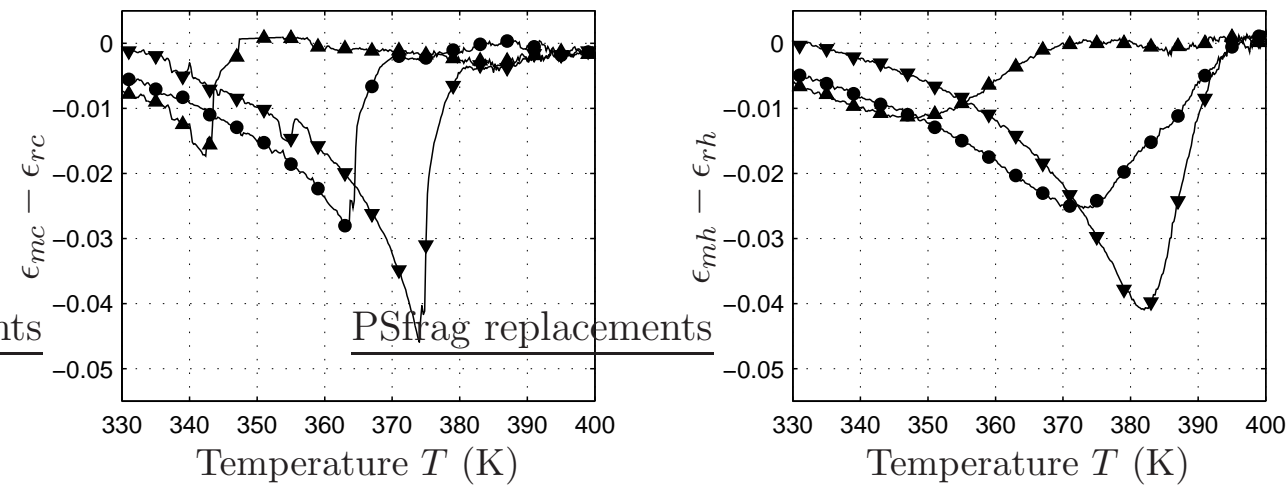

Figure 6: Dependence on $T_{\text {stop }}$. Difference between $\epsilon_{r}$ and $\epsilon_{m}$ for 3 different cooling stops measured at $\nu=0.1 \mathrm{~Hz}$ for $|R|=10 \mathrm{~K} / \mathrm{h}$ and $t_{\text {stop }}=10 \mathrm{~h}$. (a) Writing memory (cooling): $\epsilon_{m c}-\epsilon_{r c}$ with $T_{s t o p}=344 K(\mathbf{\Lambda}), 364 K(\bullet)$ and $374 K(\mathbf{v})$. (b) Reading memory (heating): $\epsilon_{m h}-\epsilon_{r h}$ (same symbols as in (a) ) .

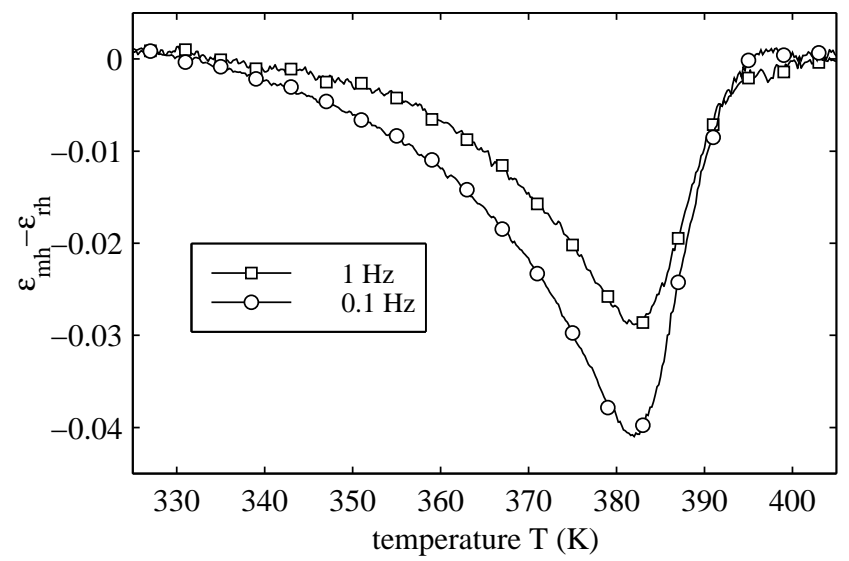

Figure 7: Dependence on frequency. Reading the memory (difference between heating curves $\epsilon_{m h}-\epsilon_{r h}$ ) after a $10 \mathrm{~h}$ stop at $T_{\text {stop }}=374 \mathrm{~K}$ during cooling. The same rate of $10 K / h$ is used but the measurement is done at different frequencies: $\nu=1 \mathrm{~Hz}(\square)$ and $\nu=0.1 \mathrm{~Hz}(\mathrm{o})$. 

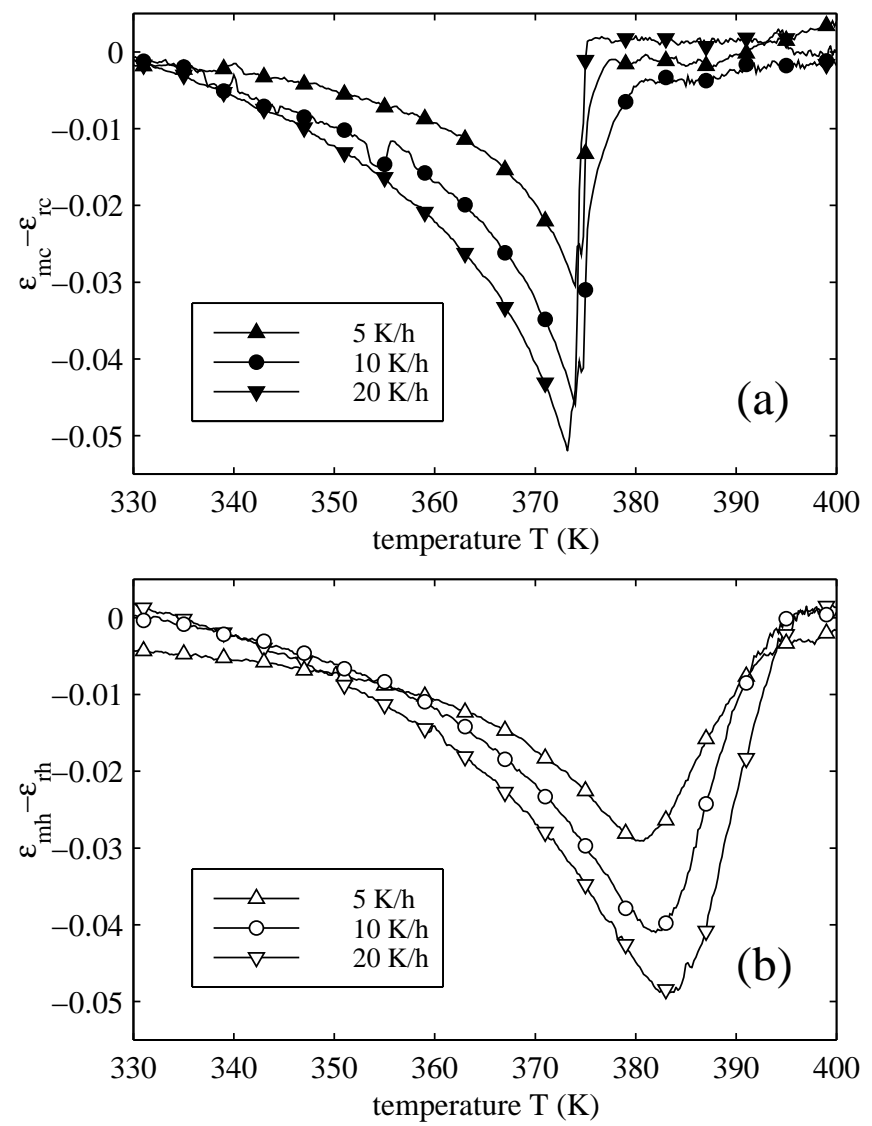

Figure 8: Dependence on the cooling and heating rate. Difference between $\epsilon_{r}$ and $\epsilon_{m}$ (aging at $T_{\text {stop }}=374 K$ for $10 h$ ) measured at $\nu=0.1 H z$ for $3|R|$. (a) Writing memory (cooling): $\epsilon_{m c}-\epsilon_{r c}$ at $5 K / h(\boldsymbol{\Delta}), 10 K / h(\bullet)$ and $20 K / h$ $(\boldsymbol{\nabla})$. (b) Reading memory (heating): $\epsilon_{m h}-\epsilon_{r h}$ at $5 K / h(\triangle), 10 K / h(\circ)$ and $20 K / h(\nabla)$. 

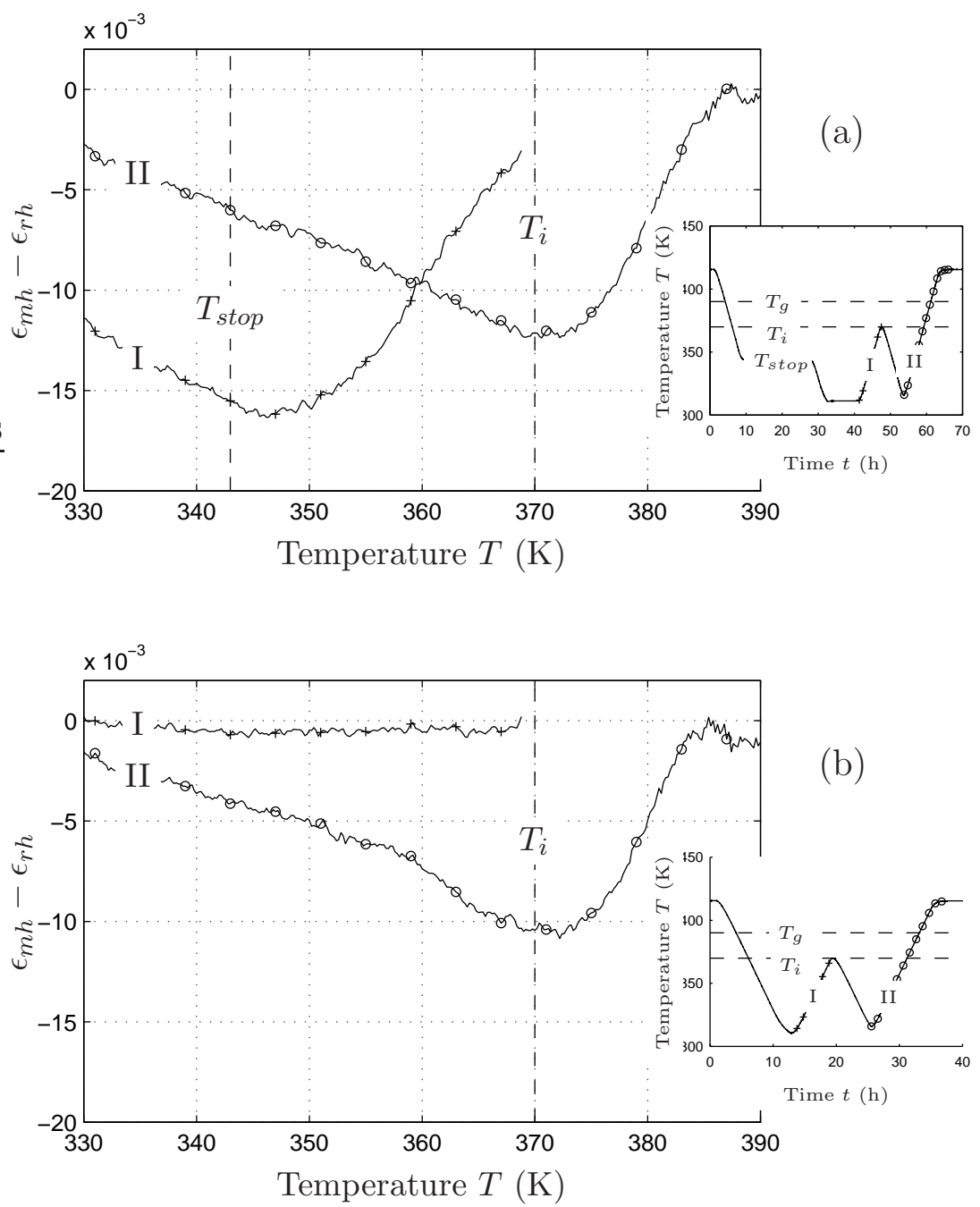

Figure 9: Deleting a memory by reading it. (a) Difference between heating curves $\epsilon_{r h}$ and $\epsilon_{m h}$ after the temperature history shown in the inset: after a classic reading the memory of a $20 h$ stop at $T_{\text {stop }}=345 \mathrm{~K}$ with $|R|=20 \mathrm{~K} / \mathrm{h}$ and $\nu=0.1 H z(+)$, the sample is cooled again when the temperature reaches $T_{i}<T_{g}$. The second reading (o) is very different since no tracks of $T_{\text {stop }}$ is found but a sort of memory of the inversion temperature $T_{i}$. This can be checked on (b), where we only test the memory of the inversion. The difference between the second heating curves (o) $\epsilon_{m h}-\epsilon_{r h}$ is exactly the same for both temperature histories. Heating the sample up to $T_{i}$ reinitializes the lower temperature behavior, even though $T_{i}<T_{g}$. 


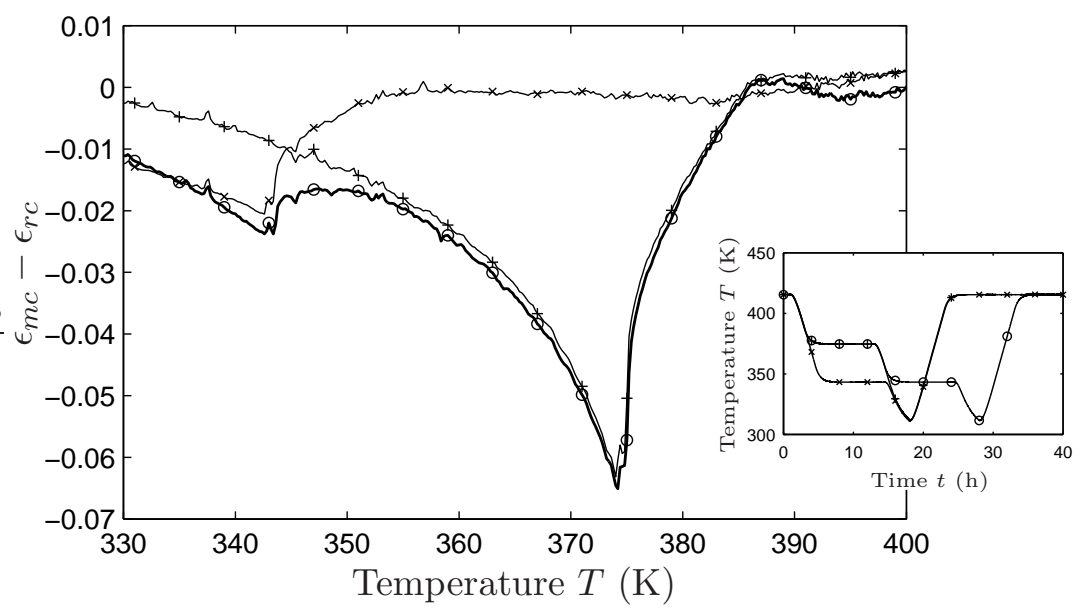

Figure 10: Double memory recording. Difference between cooling curves $\epsilon_{m c}$ and $\epsilon_{r c}$ during the temperature history shown in the inset, for $|R|=$ $20 \mathrm{~K} / \mathrm{h}$, and $\nu=0.1 \mathrm{~Hz}$ : one $10 \mathrm{~h}$ stop at $T_{\text {stop } 1}=375 \mathrm{~K}(+)$, one $10 \mathrm{~h}$ stop at $T_{\text {stop } 2}=345 \mathrm{~K}(\mathrm{x})$, and two $10 \mathrm{~h}$ stops at $T_{\text {stop } 1}=375 \mathrm{~K}$ and $T_{\text {stop } 2}=345 \mathrm{~K}$ (o). The low temperature state is independent of high temperature history: aging during $10 h$ at $T_{\text {stop } 2}$ produces the same relaxation, whatever happened before at higher temperatures. 


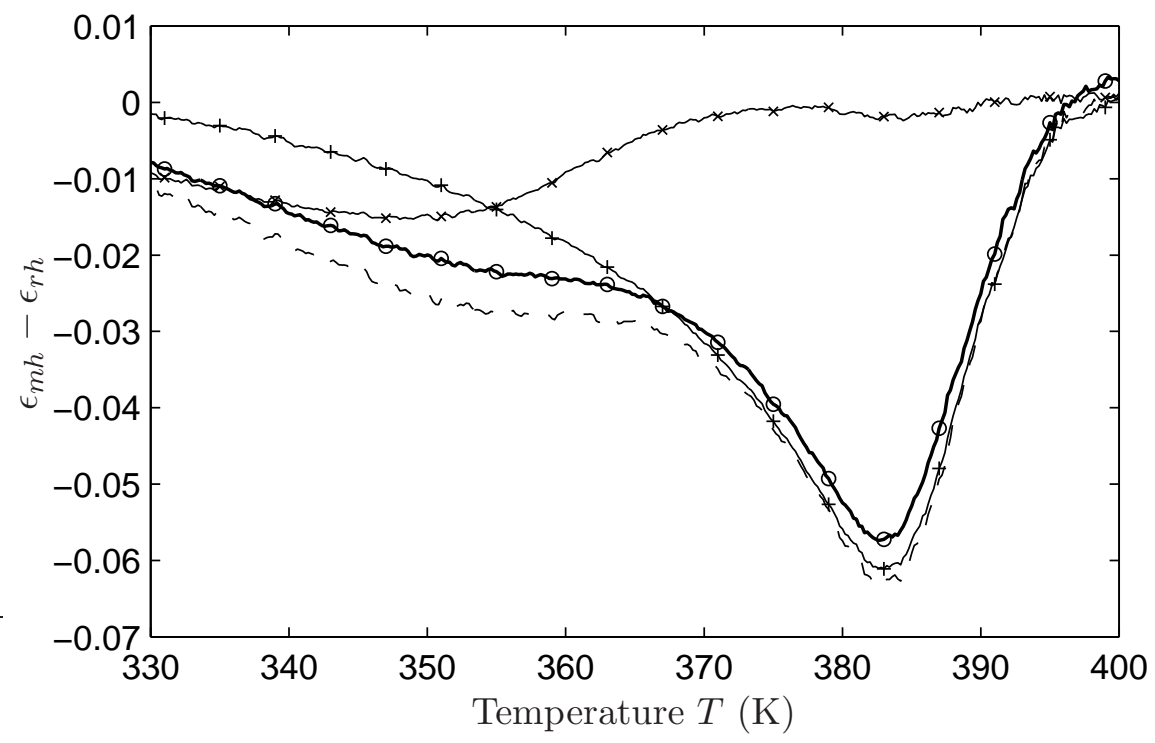

Figure 11: Double memory reading. Difference between heating curves $\epsilon_{m h}$ and $\epsilon_{r h}$ corresponding to the cooling curves of fig 10: reading of a single stop at $T_{\text {stop } 1}=375 \mathrm{~K}(+)$ and $T_{\text {stop } 2}=345 \mathrm{~K}(\mathrm{x})$, reading of a double stop at $T_{\text {stop } 1}$ and $T_{\text {stop } 2}(\mathrm{o})$. The dashed line is the sum of the two single stop curves, it is a very good approximation of the double memory reading. Memory effects thus appear only additive in this case, where $T_{\text {stop } 1}$ and $T_{\text {stop } 2}$ are sufficiently far from each other. 